\title{
Evaluation of Yield and its Components in Bread Wheat (Triticum aestivum L.) Genotypes
}

\author{
Raj Kumar ${ }^{*}$, Hadi Bux Bozdar ${ }^{2}$, Karim Dino Jamali $^{3}$ and Mehboob Ali Sial ${ }^{3}$ \\ ${ }^{1}$ Department of Botany Adamjee Government Science College, Karachi, Sindh, Pakistan \\ ${ }^{2}$ Institute of Plant Sciences, University of Sindh, Jamshoro 76080, Pakistan. \\ ${ }^{3}$ Plant Genetics Division, Nuclear Institute of Agriculture (NIA), Tando Jam- 70060, \\ Pakistan. \\ *Corresponding author: rajkumarudasi@ gmail.com. \\ Article Received 03-07-2020, Revised 28-08-2020, Accepted 12-09-2020
}

\begin{abstract}
Bread wheat is an important food crop of world and Pakistan. An experiment was conducted in winter wheat growing season to assess yield and yield related traits of newly evolved wheat genotypes. The 16 wheat genotypes includes 14 advanced lines viz., CIM-04-5, CIM-04-21, CIM-04-3, C7-98-11, 5-02, V2-1012, CIM-03-2, C2-98- 6, 6-12, V3-10-9, C6-98-5, V3-10-32, C2-98-8, V2-10-21 and 2 local checks NIA Sunhari and Kiran 95 were tested. Experimental design was laid out in RCBD with 3 replicates. Mean square for genotypes showed high significantly differences for most of agro-morphological characters. Mean and range of all wheat genotypes for all the traits indicated a considerable variability between genotypes. Mean performance for the trait grain yield showed that newly developed genotypes C2-98-8, CIM-04-21, V3-10-32 and CIM-04-3 produced higher grain yield ( 3 to $\left.3.25 \mathrm{~kg} \mathrm{plot}^{-1}\right)$ than both the contesting check varieties. High significantly and positively correlation of the plot yield to thousand grain weight $\left(0.41^{* *}\right)$, biomass $(0.41 * *)$ and harvest index $\left(0.86^{* * *}\right)$ with grain yield were found. It indicated that by improving these three traits, we can significantly improve grain yield. Selected genotypes and traits can be used in breeding program for wheat improvement.
\end{abstract}

Keywords: Bread wheat, biomass, genotypes, grain yield, harvest index, thousand grain weight

\section{Introduction}

Bread wheat (Triticum aestivum L.) has special position as compared to other cereals in Pakistan and worldwide. It is first cereal and precious agricultural product of the world. The population growth and their high consumption in advanced and developing countries to rise in the world demand for food Arzani (2004). The regularly demand to determine and develop newly wheat genotypes with resistance pressure for production of high grain yield (Riazuddin et al, 2010). A complex polygenic trait is the wheat grain yield controlled by potentiality of genotype, environment and its yield attributes viz. grain yield, harvest index, spike yield, plant height etc (Sial et al., 2000). The relationship of various yield components and environmental factors with grain yield and its related traits. Wheat genotypes with high yielding and improved traits developed through breeding techniques viz. selection, hybridization etc. Genotypes were tested at various locations to choose genotypes $\mathrm{x}$ environment interaction and asses their stability. Further, genotypes were made prone to various analytical methods viz. analysis of variance, correlation etc. for next advanced lines (Knezevic et al., 2008; Yousaf et al., 2008). Wheat yield can be improved based on related improved yield components (Ashfaq et al., 2003). The wheat improvement program is to raise grain yield that requires fruitful knowledge based on the parental materials in favor of variation for yield and its contributing characters. This is needed for breeding selection. (Khalil Ahmed Laghari, 2009; Sarfraz et al., 2020)) researched on performance of genotypes for yield and its characters with reference to agromorphological characters. Researchers were concluded that the effective selection for grain yield by agro-morphological traits and analytical methods. This study indicated the performance of yield components in advance lines and correlation among traits for grain yield to choose high yielding wheat advance lines for breeding program.

\section{MATERIAL AND METHODS}

The 16 genotypes of wheat were studied. Experiment was designed in RCBD with 3 
Kumar et al.,

replications during 2014-15 at Nuclear Institute of Agriculture (NIA), Tandojam. The total plot of $1.8 \mathrm{~m} \mathrm{x}$ $5 \mathrm{~m}=9 \mathrm{~m} 2$ and contained of six rows per genotypes with row to row 30 centimeter in distance was utilized for this trial. All the cultural practice was performed throughout the experiment. The suggested quantity of nitrogen $\left(120 \mathrm{~kg} \mathrm{ha}^{-1}\right)$, urea and phosphorus $(90 \mathrm{~kg}$ $\mathrm{P}_{2} \mathrm{O}_{5} \mathrm{ha}^{-1}$ ) and Diammonium phosphate were practiced to the wheat crop. The basal dose of phosphatic fertilizer and $1 / 3$ of nitrogen were applied during sowing time and two remaining splits of nitrogen during heading and tillering stage. Soaking irrigation was practiced, and soil was irrigated four times viz. three leaf, tillering, heading dough stage at the time of entire growing season. Ten plants randomly were selected from each genotype for recording of observations. Days to heading was observed when spikes seventy five percent emerged from flag leaf. Plant height and spike length were measured from soil surface to tip of spike excluding awn. Ten randomly collected spikelets and grains per spike and counted at maturity and harvesting period. After thresh; grains were studied for yield spike ${ }^{-1}$ averaged for main spike yield. Biomass of wheat was taken by total dry matter per plant in kilograms. Dry weight in four rows was taken yield per plot in kilograms. Thousand grains of each genotype from each replicate were assessed and weighted in grams. Harvest index (\%) was made as grains to the biological yield ratio. Harvest index $=$ Plot grain yield $/$ biological yield $=x 100$.

Data Analysis: The collected data of each trait was analyzed to mean and range, analysis of variance, DMRT and Correlation Coefficient by using statistically software Statistix 8.1 version (Steel \& Torrie, 1980; Gomez 1983).

\section{RESULTS AND DISCUSSIONS}

Mean square from analysis of variance showed all the genotypes were highly significant differences at 0.001 probability level for all the agro-morphological traits. These results concluded the high variability among genotypes for selection of traits and for grain yield. The minimum environmental effect on the grain yield might be non-significant replication. It means experiment is good.

Days to heading: Mean square disclosed that high significantly differences $(\mathrm{P}<0.01)$ among 16 lines for days to heading. It means all lines were differing from each other (Table 1). The mean (80.50), standard error (0.46) and ranges (74-87) presented diversity in wheat genotypes (Table 2). The mean performance minimum range 74 taken for C2-98-6 while, maximum (87) was taken for CIM-03-2 (Table 3). According to
J. appl. Res in Plant Sci. Vol. 2(1), 76-82 2021

www.joarps.org.

correlation result days to heading was correlated positively significant with spike length $\left(0.38^{* * *}\right)$ whereas, negative with grain yield (Table 4). It means rise in days to heading linked to significantly reduce in grain yield. Ashraf et al., (2012); Yao et al., (2014) and Farshadfar et al., (2013) reported that genotypes were significantly different for all the characters.

Plant height $(\mathbf{c m})$ : Mean square explained highly significantly differences for plant height (Table 1). It ranged from $96-104 \mathrm{~cm}$ with mean $(101.3 \mathrm{~cm})$ and standard error (0.46) (Table 2). Mean performance minimum range 96 taken for $\mathrm{V} 2-10-12$ and NIA Sunhari while, maximum (104) was taken for C6-98-5 (Table 3). The correlation coefficient of plant height was positive and non-significant with biomass, thousand grains weight, spike grain yield, grains per spike, spike length and spikelets spike ${ }^{-1}$ while, negative with grain yield (Table 4). The report of Jamali (2008) was similar that plant height as correlated negative to grain yield.

Spike length (cm): Mean square suggested the highly significant differences among 16 lines. (Table 1). Spike length ranged from $10.78-14.25 \mathrm{~cm}$ with mean $(12.62 \mathrm{~cm})$ and standard error $(0.27)$ for spike length (Table 2). The mean performance declared lowest range 11 for 5-02 and V2-10-12 while, highest (14) was taken for V3-10-9 and CIM-03-2 (Table 3). The correlation results revealed spike length was positive and highly significant with grains spike $^{-1}\left(0.58^{* * *}\right)$, spike yield $\left(0.38^{* * *}\right)$ and spikelets spike ${ }^{-1}\left(0.55^{* * *}\right)$ whereas, negative with grain yield (Table 4). Adnan et al., (1994), Ansari et al., (1997) and, Ul-haq et al., (2010) and Jamali et al., (2003) were suggested that spike length was highly significant and positively correlated with same traits.

Spikelets/spike: Mean square outcomes specified that genotypes were highly significant different (Table 1). The ranged from 17.40-23.60 with mean value (19.66) and standard error (0.34) among all the 16 wheat genotypes (Table 2). The DMRT indicated that highest number (23.60) of spikelets/spike was found in V3-10-9 and lowest (17.40) in advance line 5-02 (Table 3). Spikelets/spike was made positive and significantly correlation with grains/spike $\left(0.48^{* * *}\right)$ while, negative with grain yield (Table 4). Findings were related of Tazeene et al., (2009), Narwal et al, (1999) and Kumar et al. (2013).

Grains/spike: The result of mean square declared that highly significant differences which reflect variation in genotypes (Table 1). The grains/spike was ranged from 45-66 with mean (57) and standard error (1.20) (Table 2). Mean performance suggested that mean differences in between genotypes as highest grains per 
spike in V3-10-9 (66) and CIM-03-2 (62) while, lowest in 5-02 (45) (Table 3). Grains/spike had highly significant and positive correlation with spike yield $(0.73 * * *)$ while, non-significant and negative with grain yield (Table 4). Findings of Eid, (2009) were same that explained grains/spike was positive correlation with spike yield.

Spike yield (g): Mean square explained that genotypes are at highly significant level (Table 1). It was ranged between 1.69 to $2.69 \mathrm{~g}$ with mean $(2.23 \mathrm{~g}$ ) and error (0.06) among all wheat genotypes (Table 2). The mean performance was noted highest grain yield in CIM-04-21 (2.69g) and CIM-04-3 (2.67g) whereas, lowest in C7-98-11 (1.69g) line (Table 3). The correlation of spike yield was positive and significant with thousand grain weight $\left(0.31^{* *}\right)$ whereas, nonsignificant with grain yield (Table 4). It means both traits had positive correlation with grain yield. Wang et al., (1991) were declared same findings.

Biomass (kg): All genotypes were highly at mean square level for biomass (Table 1). It is varied from 4.90 to $6.50 \mathrm{~kg}$ with mean and standard error $(5.97 \mathrm{~kg}$ \& 0.09) (Table-2). Mean differences showed that maximum biomass was noted in CIM-04-3 and C298-8 (6.40-6.50kg) while, the minimum in $\mathrm{V} 3-10-9$ $(4.90 \mathrm{~kg})$ genotype (Table 3$)$. Biomass was made significant and positive correlation with gain yield $\left(0.41^{* *}\right)$ and thousand grain weight $(0.37 * *)$ (Table 4). (Aruna \& Raghaviah, 1997; Giunta et al., 1999) and Singh (2001) were expressed the same findings.

Plot Grain Yield (kg): Genotypes were highly significantly different for grain yield (Table 1). It varied (1.55 to $3.25 \mathrm{~kg})$, mean $(2.72 \mathrm{~kg})$ and error

Table 2. Range and Mean Performance of sixteen advance lines including two check varieties for agromorphological traits studied during 2014- 2015

\begin{tabular}{|c|c|c|c|c|}
\hline \multirow[t]{2}{*}{ Traits } & \multirow{2}{*}{ Mean \pm S. E } & \multicolumn{2}{|l|}{ Range } & \multirow[b]{2}{*}{ Variance } \\
\hline & & Min. & Min. & \\
\hline Days to heading & $80.50 \pm 0.46$ & 74.33 & 87 & 11.89 \\
\hline Plant height $(\mathrm{cm})$ & $101.37 \pm 0.46$ & 96.79 & 104.47 & 3.35 \\
\hline Spike length $(\mathrm{cm})$ & $12.62 \pm 0.27$ & 10.87 & 14.25 & 1.20 \\
\hline Spikelets spike ${ }^{-1}$ & $19.66 \pm 0.34$ & 17.40 & 23.60 & 1.82 \\
\hline Grains spike $^{-1}$ & $57.37 \pm 1.20$ & 45.33 & 66.23 & 23.09 \\
\hline Spike yield (gram) & $2.23 \pm 0.06$ & 1.69 & 2.69 & 0.07 \\
\hline Biomass (kg) & $5.95 \pm 0.09$ & 4.90 & 6.50 & 0.15 \\
\hline Plot grains yield $(\mathrm{kg})$ & $2.73 \pm 0.10$ & 1.55 & 3.25 & 0.15 \\
\hline Harvest index (\%) & $45.61 \pm 1.20$ & 31.63 & 50.32 & 23.29 \\
\hline Thousand grain weight (gram) & $41.24 \pm 1.05$ & 34.92 & 51.01 & 17.45 \\
\hline
\end{tabular}

*S.E: Standard Error
(0.10) (Table-2). The mean differences from mean performance that higher grain yield in V3-10-32, CIM-04-21, 04-3 and C2-98-8 (3.02-3.25 kg) whereas, lower in V3-10-9 (1.55 kg) genotypes (Table 3). Grain yield was highly significantly and positive correlation with thousand grain weight $\left(0.41^{* *}\right)$ and harvest index (0.86***) (Table 4). Slafer et al., (1991) and Reynolds et al., (1999) proposed rise in grain yield could be linked with increase in harvest index.

Harvest Index (\%) The result of mean square was introduced highly significant differences for harvest index (Table 1). It was made range between 31.63$50.32 \%$ with mean $45.61 \%$ and 1.20 standard error levels (Table-2). The mean performance result was observed as higher in V3-10-32 (50.32\%) and CIM04-3 (50.00\%) while, lower in V3-10-9 (31.63\%) genotypes (Table-3). Harvest index was high significantly and positive correlation with grain yield $\left(0.86^{* * *}\right)$ and thousand grain weight $\left(0.30^{* * *}\right)$ (Table-4). The research of Singh and Sharma (1994) were same finding.

Thousand grain weight: Genotypes were highly significant different for thousand grain weight at mean square level (Table 1). It was varied (34.92-51.01g), mean and error $(41 \mathrm{~g} \pm 1.05)$ level (Table 2). The increased weight in CIM-04-21 (47g) and CIM-04-3 (51g) while, decreased in V3-10-9 (34.92g) line (Table 3). Thousand grain weight was made significant and positive correlation with biomass $(0.37 * *)$, harvest index $\left(0.30^{* * *}\right)$, grain yield $(0.41 * *)$ and spike yield $(0.31 * *)$ (Table 4$)$. Similar result conforms of Fuma et al., (2005).

S. E: Standard Error 
Table 1. Mean square of the 10 traits of 16 wheat genotypes from analysis of variance

\begin{tabular}{|c|c|c|c|c|c|c|c|c|c|c|c|}
\hline \multirow{2}{*}{$\begin{array}{l}\text { Source of } \\
\text { variation }\end{array}$} & \multicolumn{11}{|c|}{ Mean squares } \\
\hline & D.F & $\begin{array}{l}\text { Days to } \\
\text { heading }\end{array}$ & $\begin{array}{l}\text { Plant } \\
\text { height } \\
(\mathrm{cm})\end{array}$ & $\begin{array}{l}\text { Spike } \\
\text { length } \\
(\mathrm{cm})\end{array}$ & $\begin{array}{l}\text { Spikelet } \\
\text { Spike }^{-1}\end{array}$ & $\begin{array}{l}\text { Grains } \\
\text { spike }^{-1}\end{array}$ & $\begin{array}{l}\text { Spike } \\
\text { yield (gm) }\end{array}$ & $\begin{array}{l}\text { Biomass } \\
\text { (kg) }\end{array}$ & $\begin{array}{l}\text { Grain } \\
\text { yield } \\
\text { (kg) }\end{array}$ & $\begin{array}{l}\text { Harvest } \\
\text { Index }(\%)\end{array}$ & $\begin{array}{l}\text { TGW } \\
\text { (gm) }\end{array}$ \\
\hline Replications & 2 & 1.458 & 31.902 & 4.4380 & $55.577 \mid$ & 54.808 & $\mathrm{I} .09874$ & 3.48958 & 0.09115 & 44.603 & 96.768 \\
\hline Genotypes & 15 & $\begin{array}{l}325.986 \\
* * *\end{array}$ & $\begin{array}{l}134.586 \\
* * *\end{array}$ & $\begin{array}{l}38.3587 \\
* * *\end{array}$ & $\begin{array}{l}67.9221 \\
* * *\end{array}$ & $\begin{array}{l}716.764 \\
* * *\end{array}$ & $\begin{array}{l}2.28188 \\
* * *\end{array}$ & $\begin{array}{l}7.27778 \\
* * *\end{array}$ & $\begin{array}{l}5.29319 \\
* * *\end{array}$ & $\begin{array}{l}521.302 \\
* * *\end{array}$ & $\begin{array}{l}526.55 \\
* * *\end{array}$ \\
\hline Error & 462 & 0.210 & 5.445 & 1.0638 & 3.1169 & 100.129 & 0.27090 & 0.08230 & 0.04409 & 5.965 & 1.718 \\
\hline Total & 479 & & & & & & & & & & \\
\hline L.S.D (0.05) & & 0.2326 & 1.1839 & 0.5233 & 0.8958 & 5.0772 & $0.264 I$ & 0.1456 & 0.1065 & 1.2392 & 0.6650 \\
\hline
\end{tabular}

$* * *$ Highly Significant at the 0.001 respectively

Table-3 Mean performance for agro-morphological traits in wheat genotypes

\begin{tabular}{|c|c|c|c|c|c|c|c|c|c|c|c|}
\hline Sr. & Genotypes & $\begin{array}{l}\text { Days to } \\
\text { heading }\end{array}$ & $\begin{array}{l}\text { Plant } \\
\text { Height }(\mathbf{c m})\end{array}$ & $\begin{array}{l}\text { Spike } \\
\text { Length } \\
(\mathrm{cm})\end{array}$ & $\begin{array}{l}\text { Spikelet } \\
\text { Spike }^{-1}\end{array}$ & $\begin{array}{l}\text { Grains } \\
\text { Spike }^{-1}\end{array}$ & $\begin{array}{l}\text { Spike } \\
\text { Yield } \\
\text { (gram) }\end{array}$ & $\begin{array}{l}\text { Bio- } \\
\text { mass } \\
(\mathrm{Kg})\end{array}$ & $\begin{array}{l}\text { Grain } \\
\text { Yield. } \\
(\mathbf{K g})\end{array}$ & $\begin{array}{l}\text { Harvest } \\
\text { Index } \\
(\%)\end{array}$ & $\begin{array}{l}1000 \\
\text { Grain } \\
\text { Weight } \\
\text { (gram) }\end{array}$ \\
\hline 1. & CIM-04-21 & $81.00 \mathrm{G}$ & $100.53 \mathrm{FG}$ & $13.20 \mathrm{~B}$ & $19.43 \mathrm{DE}$ & 56.07 DEFG & $2.67 \mathrm{~A}$ & $6.20 \mathrm{~B}$ & $3.02 \mathrm{BC}$ & $48.70 \mathrm{~B}$ & $47.33 \mathrm{~B}$ \\
\hline 2. & CIM-04-3 & $83.00 \mathrm{E}$ & $100.27 \mathrm{FG}$ & $13.13 \mathrm{~B}$ & $20.17 \mathrm{BCD}$ & $54.87 \mathrm{EFG}$ & $2.69 \mathrm{~A}$ & $6.50 \mathrm{~A}$ & $3.25 \mathrm{~A}$ & $50.00 \mathrm{~A}$ & $51.01 \mathrm{~A}$ \\
\hline 3. & CIM-04-5 & $84.00 \mathrm{~B}$ & $101.20 \mathrm{EF}$ & $13.02 \mathrm{BC}$ & $20.50 \mathrm{~B}$ & $58.43 \mathrm{BCDEF}$ & $2.53 \mathrm{AB}$ & $5.50 \mathrm{~F}$ & $2.45 \mathrm{G}$ & $44.54 \mathrm{GH}$ & $42.06 \mathrm{E}$ \\
\hline 4. & 6-12 & $82.33 \mathrm{~F}$ & 102.17 CDE & $12.35 \mathrm{DE}$ & $20.33 \mathrm{BC}$ & $60.93 \mathrm{BCD}$ & $2.06 \mathrm{DE}$ & $5.80 \mathrm{D}$ & $2.60 \mathrm{~F}$ & $44.82 \mathrm{GH}$ & $36.33 \mathrm{~J}$ \\
\hline 5. & $5-02$ & $79.67 \mathrm{H}$ & 102.50 BCD & $11.00 \mathrm{G}$ & $17.40 \mathrm{H}$ & $45.33 \mathrm{H}$ & $1.90 \mathrm{EF}$ & $5.90 \mathrm{C}$ & $2.23 \mathrm{H}$ & $37.79 \mathrm{I}$ & $44.27 \mathrm{C}$ \\
\hline 6. & V2-10-12 & $79.67 \mathrm{H}$ & $96.79 \mathrm{H}$ & $11.05 \mathrm{G}$ & $19.06 \mathrm{EF}$ & $53.50 \mathrm{FG}$ & $1.99 \mathrm{E}$ & $6.00 \mathrm{BC}$ & $2.81 \mathrm{D}$ & $46.83 \mathrm{CD}$ & $39.15 \mathrm{H}$ \\
\hline 7. & CIM-03-2 & $87.00 \mathrm{~A}$ & $101.90 \mathrm{DE}$ & $14.07 \mathrm{~A}$ & $19.13 \mathrm{EF}$ & $62.83 \mathrm{AB}$ & $2.29 \mathrm{BCD}$ & $6.20 \mathrm{~B}$ & $2.81 \mathrm{D}$ & $45.32 \mathrm{EF}$ & $38.13 \mathrm{I}$ \\
\hline 8. & C2-98-6 & $74.67 \mathrm{M}$ & $101.37 \mathrm{EF}$ & $13.30 \mathrm{~B}$ & $20.33 \mathrm{BC}$ & $61.63 \mathrm{ABC}$ & $2.35 \mathrm{BC}$ & $5.80 \mathrm{D}$ & $2.72 \mathrm{DE}$ & $46.89 \mathrm{CD}$ & $43.16 \mathrm{D}$ \\
\hline 9. & V2-10-21 & $78.67 \mathrm{I}$ & $99.77 \mathrm{G}$ & $12.60 \mathrm{CD}$ & $17.63 \mathrm{H}$ & $58.43 \mathrm{BCDEF}$ & $2.35 \mathrm{BC}$ & $6.30 \mathrm{~B}$ & $2.98 \mathrm{C}$ & $47.30 \mathrm{BC}$ & $42.17 \mathrm{E}$ \\
\hline 10. & V3-10-9 & $83.67 \mathrm{C}$ & $102.00 \mathrm{DE}$ & $14.25 \mathrm{~A}$ & $23.60 \mathrm{~A}$ & $66.23 \mathrm{~A}$ & $2.12 \mathrm{CDE}$ & $4.90 \mathrm{G}$ & $1.55 \mathrm{I}$ & $31.63 \mathrm{~J}$ & $34.92 \mathrm{~K}$ \\
\hline 11. & C6-98-5 & $76.33 \mathrm{~L}$ & $104.47 \mathrm{~A}$ & $12.07 \mathrm{EF}$ & $20.30 \mathrm{BCD}$ & $58.90 \mathrm{BCDE}$ & $2.15 \mathrm{CDE}$ & $5.90 \mathrm{C}$ & $2.62 \mathrm{EF}$ & $44.40 \mathrm{FG}$ & $36.44 \mathrm{~J}$ \\
\hline 12. & C7-98-11 & $83.33 \mathrm{D}$ & $103.20 \mathrm{BC}$ & $13.35 \mathrm{~B}$ & $20.60 \mathrm{~B}$ & $53.60 \mathrm{FG}$ & $1.69 \mathrm{~F}$ & $5.70 \mathrm{E}$ & $2.62 \mathrm{EF}$ & $45.96 \mathrm{EF}$ & $39.21 \mathrm{H}$ \\
\hline 13. & C2-98-8 & $77.67 \mathrm{~J}$ & $102.87 \mathrm{BCD}$ & $10.98 \mathrm{G}$ & $18.70 \mathrm{EFG}$ & $60.33 \mathrm{BCD}$ & $2.37 \mathrm{BC}$ & $6.40 \mathrm{~B}$ & $3.12 \mathrm{~B}$ & $48.75 \mathrm{~B}$ & $41.20 \mathrm{~F}$ \\
\hline 14. & V3-10-32 & $77.33 \mathrm{~K}$ & 102.20 CDE & $11.80 \mathrm{~F}$ & $18.03 \mathrm{GH}$ & 57.57 CDEFG & $2.37 \mathrm{BC}$ & $6.20 \mathrm{~B}$ & $3.12 \mathrm{~B}$ & $50.32 \mathrm{~A}$ & $42.58 \mathrm{DE}$ \\
\hline 15. & Nia Sunhari & $78.67 \mathrm{I}$ & $96.80 \mathrm{H}$ & $10.78 \mathrm{G}$ & $19.53 \mathrm{CDE}$ & $53.00 \mathrm{G}$ & $1.97 \mathrm{E}$ & $5.92 \mathrm{C}$ & $2.98 \mathrm{C}$ & $50.32 \mathrm{~A}$ & $38.220 \mathrm{I}$ \\
\hline \multirow[t]{3}{*}{16.} & Kiran 95 & $78.66 \mathrm{I}$ & $100.70 \mathrm{FG}$ & $13.41 \mathrm{~B}$ & 18.27 FGH & $59.67 \mathrm{BCDE}$ & $2.26 \mathrm{CD}$ & $6.10 \mathrm{BC}$ & $2.82 \mathrm{D}$ & $46.22 \mathrm{DE}$ & $40.26 \mathrm{G}$ \\
\hline & Mean & 80.50 & 101.37 & 12.62 & 19.66 & 57.37 & 2.23 & 5.95 & 2.73 & 45.61 & 41.24 \\
\hline & L.S.D (0.05) & 0.2326 & 1.1839 & $\mathbf{0 . 5 2 3 3}$ & 0.8958 & 5.0772 & 0.2641 & 0.1456 & 0.1065 & 1.2392 & 0.6650 \\
\hline
\end{tabular}


www.joarps.org.

Table 4. Scatter (Simple) Correlation coefficient among agro-morphological traits of 16 wheat genotypes

\begin{tabular}{|c|c|c|c|c|c|c|c|c|c|}
\hline Traits & $\begin{array}{c}\text { Plant } \\
\text { height } \\
(\mathrm{cm})\end{array}$ & $\begin{array}{c}\text { Spike } \\
\text { length } \\
\text { (cm) }\end{array}$ & $\begin{array}{c}\text { Spikelets } \\
\text { spike }^{-1}\end{array}$ & $\begin{array}{l}\text { Grains } \\
\text { spike }^{-1}\end{array}$ & $\begin{array}{c}\text { Main } \\
\text { spike } \\
\text { yield } \\
\text { (gram) }\end{array}$ & $\begin{array}{c}\text { Biomass } \\
\text { (kg) }\end{array}$ & $\begin{array}{c}\text { Plot } \\
\text { grain } \\
\text { yield }(\mathbf{k g})\end{array}$ & $\begin{array}{c}\text { Thousan } \\
\text { d grain } \\
\text { weight } \\
\text { (gram) }\end{array}$ & $\begin{array}{c}\text { Harvest } \\
\text { index } \\
(\%)\end{array}$ \\
\hline Days to heading & $0.07^{\mathrm{NS}}$ & $0.38^{* * *}$ & $0.21^{\mathrm{NS}}$ & $0.046^{\mathrm{NS}}$ & $-0.01^{\mathrm{NS}}$ & $0.05^{\mathrm{NS}}$ & $-0.33^{* *}$ & $-0.06^{\mathrm{NS}}$ & $-0.31^{* *}$ \\
\hline Plant height $(\mathrm{cm})$ & & $0.17^{\mathrm{NS}}$ & $0.03^{\mathrm{NS}}$ & $0.03^{\mathrm{NS}}$ & $0.04^{\mathrm{NS}}$ & $0.13^{\mathrm{NS}}$ & $-0.18^{\mathrm{NS}}$ & $0.10^{\mathrm{NS}}$ & $-0.18^{\mathrm{NS}}$ \\
\hline Spike length $(\mathrm{cm})$ & & & $0.55^{* * *}$ & $0.58^{* * *}$ & $0.38^{* * *}$ & $-0.02^{\mathrm{NS}}$ & $-0.30^{* *}$ & $-0.03^{\mathrm{NS}}$ & $-0.25^{*}$ \\
\hline No. of Spikelets spike ${ }^{-1}$ & & & & $0.48^{* * *}$ & $0.20^{\mathrm{NS}}$ & $-0.30^{* *}$ & $-0.32^{* *}$ & $-0.24^{*}$ & $-0.29^{*}$ \\
\hline No. of grains spike ${ }^{-1}$ & & & & & $0.73^{* * *}$ & $-0.07^{\mathrm{NS}}$ & $-0.10^{\mathrm{NS}}$ & $-0.15^{\mathrm{NS}}$ & $-0.10^{\mathrm{NS}}$ \\
\hline Main spike yield (gram) & & & & & & $0.16^{\mathrm{NS}}$ & $0.22^{\mathrm{NS}}$ & $0.31^{* *}$ & $0.13^{\mathrm{NS}}$ \\
\hline Biomass (kg) & & & & & & & $0.41^{* *}$ & $0.37^{* *}$ & $0.07^{\mathrm{NS}}$ \\
\hline Plot grain yield $(\mathrm{kg})$ & & & & & & & & $0.41^{* *}$ & $0.86^{* * *}$ \\
\hline 1000 grain weight (gram) & & & & & & & & & $0.30^{* * *}$ \\
\hline
\end{tabular}

* Significant at the 0.5 .

** Highly Significant at the 0.01;

*** Highly Significant at the 0.001 respectively;

NS Non-Significant; 
Kumar et al.,

\section{CONCLUSION}

The present research suggested high variability among 16 genotypes for selection of various characters for grain yield. The great variability provided a valuable opportunity for improvement of grain yield. Maximum grain yield $(3-3.25 \mathrm{~kg})$ was found in CIM-04-21, 04-3, C2-98-8 and V3-10-32 genotypes as compared to all the rest of lines including checks NIA-Sunhari and Kiran-95 might be medium and early days to heading, normal plant height, increased thousand grain weight, biomass, harvest index, spike yield, greater grains spike ${ }^{-1}$. The lowest yield was found in $\mathrm{V} 3-10-9(1.55 \mathrm{~kg})$. Among these traits, thousand grain weight, harvest index and biomass had strong positive performance towards grain yield. This study is selected the high yielding lines for breeding program.

\section{References:}

Adnan M, Chowdhry M.A, Mahmood T. (1994). Association of flag leaf area and spike characteristics with grain yield in aestivum spp. Pak. J. Agric. Sci., 31(1): 51-53.

Ansari B.A, Malik A.J, Larik A.S, Ansari K.A. (1997). Interdependence of yield and its components in the hybrids of Triticum aestivum L. Pak. J. Agric., Agril. Engg., Vet. Sci., 13(2): 19-20.

Aruna, C. and P. Raghavaiah. (1997). Correlation and path analysis of yield and quality in aestivum wheat (Triticum aestivum L.). J. Res., 25: 21-25.

Arzani. A., 2004. modified crops. (Translation). Publication IUT.

Asharf. A., A.E. Mohsen, S.R.A. Hegazy and M.H. Taha (2012). Genotypic and phenotypic interrelationships among yield and yield components in Egyptian bread wheat genotypes. Journal of Plant Breeding and Crop Science 4(1): 9-16.

Ashfaq, M., A.S. Khan and Z. Ali. (2003). Association of morphological traits with grain yield in wheat (Triticum aestivum L.). Int. J. Agric. Biol., 5:264-267.

Eid, M.H. (2009). Estimation of heritability and genetic advance of yield traits in wheat (Tritium aestivum L.) under drought
J. appl. Res in Plant Sci. Vol. 2(1), 76-82 2021

www.joarps.org.

condition. International Journal of Genetics and Molecular Biology, 1(7): 115-120.

Farshadfar E, Romena H, Shabani A. (2013). Evaluation of genetic parameters in agro- physiological traits of wheat (Triticum aestivum L.) under irrigated condition. International journal of Advanced Biological and Biomedical Research 1(4): 331-340.

Fuma, H., P. Stephen Baenziger, B. S. Beecher, R. A. Graybosch, K. M. Eskridge, and L. A. Nelson. (2005). Genetic improvement trends in agronomic performances and en-use quality characteristics among hard red winter wheat cultivars in ebraska. Euphytica 144: 187-198.

Giunta, F., R. Motzo and M. Deidda. (1999). Grain yield analysis of a triticale ( $\mathrm{x}$ Triticosecale Wittmack) collection grown in a Mediterranean environment. Field Crops Res., 63:199-210.

Jamali, K.D. and S.A. Ali. (2003). Exploitation of semi-dwarf wheat (Triticum aestivum L.) genotypes for high yield. Proceedings of 10th International wheat Genetics Symposium (1-6, September 2003) at Paestum, Italy, 727-729.

Jamali, K.D. and A.A. Syed. 2008. Yield and yield components with relation to plant height in semi-dwarf wheat. Pak. J. Bot., 40(4): 1805-1808.

Knezevic, M., L. Ranogajec and D. Samota, 2008. Effects of soil tillage and herbicides on weeds and winter wheat yields. Cereals Research Communications, 36 (Suppl.): 14031406.

Khalil Ahmed Laghari, Mahboob Ali Sial, M. Afzal Arain, M. Umar Dahot, M. Sher Mangrio and A.J. Pirzada. 2010. Comparative Performance of Wheat Advance Lines for Yield and its Associated Traits. IDOSI Publications. World Applied Sciences Journal 8 (Special Issue of 
Biotechnology and Genetic Engineering): 34-37.

Kumar, S., V. K. Dwivedi and N. K. Tyagi. (2003). Genetic variability in some metric traits and its contribution to yield in wheat (Triticum aestivum L.). Progressive Agric., 3(1-2): 152- 153.

Narwal N.K, Verma P.K, Narwal M.S (1999). Genetic variability, correlation and path coefficient analysis in bread wheat in two climatic zones of Hayrana. Agric. Sci. Dig. Karnal. 19(2): 73-76.

Reynolds, M.P., S. Rajaram, and K.D. Sayre. (1999). Physiological and genetic changes of irrigated wheat in the postgreen revolution period and approaches for meeting projected global demand. Crop Sci. 39:1611-1621.

Riaz-ud-din, G.M. Subhani, N. Ahmed, M. Hussain and A. Rehman. (2010). Effect of temperature on development and grain formation in spring wheat. Pak. J. Bot., 42(2): 899-906.

Sarfraz, Z., Shah, M. M., Iqbal, M. S., Nazir, M. F., \& Fatima, S. A. (2020). Cause and effect relationship of morphological attributes with yield in a-genome Wheat. Journal of Applied Research in Plant Sciences, 1(1):13-19.

Slafer, G.A., and F.H. Andrade. (1991). Changes in physiological attributes of the dry matter economy of bread wheat (Triticum aestivum L.) through genetic improvement of grain yield potential at dif- ferent regions of the world. A review. Euphytica 58:37-49.

Sing, I. and S.K. Sharma. (1994). Interrelationship of harvest index and other traits in wheat. Haryana Agric. Univ. J. Res. 24(1): 33-38.

Singh, S.B. and T.B. Singh. (2001). Correlation and path analysis in common wheat (Triticum aestivum L.) under light texture soil. G.B.P.U.A. and T. Res. Station, Ujhani-243 (UP), India. Res. Crops, 2: 99-101.
Tazeen M, Naqvi FN. (2009). Heritability, phenotypic correlation and path coefficient studies for some agronomic characters in synthetic elite lines of wheat. Journal of Food Agriculture and Environment 7(3-4): 278-282.

Ul-haq W, Munir M, Akram Z 2010. Estimation of Interrelationships among yield and yield related attributes in wheat lines. Pak. J. Bot., 42(1): 567-573.

Wang, S., Z.C. Xu, R.S. Xie and Z.H. Zhang, 1991. Comparison on the inheritance of main traits in wheat grown in south and north areas. Acta Agriculture Universitatis Henanensis, China, 25: 125:133.

Yousaf, A., B.M. Atta, J. Akhtar, P. Monneveux and Z. lateef, 2008. Genetic variability, association and diversity studies in wheat (Triticum aestivum L.) germplasm. Pak. J. Bot., 40(5): 20872093.

Yao. J. M. Hongxiang, X. Yang, G.U. Yao and M. Zhou (2014). Inheritance of grain yield and its correlation with yield components in bread wheat (Triticum aestivum L.). 13(12):1379-1385 